\title{
Defeitos de esmalte não fluoróticos em crianças: aspectos clínicos e epidemiológicos
}

Non-fluorotic enamel defects in children: clinical and epidemiological aspects

\author{
Mabel Miluska Suca Salas* \\ Luiz Alexandre Chisini* \\ Vitoria da Silva Castanheira*** \\ Ingrid Santos Castro*** \\ Luiza Souza Teixeira*** \\ Flávio Fernando Demarco ${ }^{* * *}$
}

\section{Resumo}

Objetivo: analisar as características clínicas e epidemiológicas de defeitos de desenvolvimento de esmalte em crianças com dentição permanente e decídua. Revisão de literatura: defeitos de desenvolvimento de esmalte não fluoróticos são falhas produzidas na estrutura interna do esmalte durante seu desenvolvimento. Defeitos de desenvolvimento de esmalte podem ser encontrados em ambas as dentições, com prevalências que podem variar de $23,9 \%$ a $90,4 \%$ na dentição decídua e de $52 \%$ a $92,1 \%$ na permanente. Esses distúrbios no esmalte podem estar relacionados a diversos fatores relacionados às características maternas ou das próprias crianças. A presença de defeitos de desenvolvimento de esmalte pode ser um indicativo de acontecimentos patológicos passados. Dependendo da severidade, esses defeitos podem predispor os dentes a outros problemas, como cárie ou desgaste. Se o defeito for severo, problemas estéticos dentários e consequentes problemas psicológicos podem interferir na qualidade de vida da criança. Defeitos severos podem levar à busca de tratamentos estéticos mais invasivos, promovendo perda estrutural dentária precoce. Considerações finais: $o$ conhecimento da etiologia e das características clínicas dos defeitos de desenvolvimento do esmalte é indispensável para a formulação de um plano de tratamento adequado.

Palavras-chave: Defeitos de desenvolvimento de esmalte. Dentição decídua. Dentição permanente. Fatores associados.

\section{Introdução}

Os defeitos de desenvolvimento de esmalte não fluoróticos (DDEs) estão associados a distúrbios nos estágios de formação do esmalte. Durante o desenvolvimento das estruturas dentais, uma série de fatores pode modificar a função ameloblástica, levando ao desenvolvimento de defeitos na qualidade ou quantidade de esmalte ${ }^{1}$.

A presença de defeitos de desenvolvimento de esmalte, tanto na dentição decídua quanto na permanente, pode ter uma frequência de até $90 \%$ nas crianças $^{2}$. Em crianças, o tipo de DDE e a severidade podem estar relacionados a diversos fatores ${ }^{3,4}$. $\mathrm{Na}$ dentição decídua, a literatura mostra a existência de associação entre a presença de defeitos de esmalte e alguns fatores, geralmente traumáticos, relacionados com a gestação e a ocorrência de doenças, traumas ou consumo de medicamentos na infância ${ }^{2,5,6}$. A ocorrência de problemas pré-natais e neonatais pode aumentar o risco de presença de defeitos de esmalte em crianças $^{2,5-8}$. Na dentição permanente, traumas na dentição decídua são associados à presença de defeitos de esmalte ${ }^{9-11}$, assim como à experiência de cárie na dentição decídua ${ }^{12-16}$ e à ocorrência de doenças sistêmicas na infância ${ }^{17-19}$.

A presença de defeitos hipoplásicos tem relação com predisposição à cárie ${ }^{16,20}$ e à sensibilidade den-

\footnotetext{
Pós-doutoranda em Odontologia, Universidade Federal de Pelotas. Professora da Faculdade de Ciências do Tocantins, Brasil.

Mestrando em Odontologia, Universidade Federal de Pelotas, Brasil.

* Acadêmicas de Odontologia, Universidade Federal de Pelotas, Brasil.

**** Professor titular do Programa de Pós-Graduação em Odontologia e Epidemiologia, Universidade Federal de Pelotas, Brasil.
} 
tária, maloclusão e problemas estéticos, que podem promover problemas de ordem social e psicológica, interferindo na qualidade de vida das crianças ${ }^{21}$. A etiologia dos defeitos de esmalte está geralmente relacionada com distúrbios neonatais ou pós-natais de diversas índoles, permitindo o reconhecimento da história clínica sistêmica passada do indivíduo, funcionando como um marcador de eventos ocorridos na vida.

O objetivo da presente revisão é analisar as características clínicas e epidemiológicas dos defeitos de desenvolvimento de esmalte não hereditários em crianças com dentição permanente e decídua.

\section{Revisão da literatura}

\section{Conceito}

Defeitos de desenvolvimento de esmalte de tipo não hereditários são falhas produzidas na estrutura interna do esmalte durante seu desenvolvimento ${ }^{7}$. O desenvolvimento das alterações dentárias está associado a distúrbios durante os estágios de desenvolvimento do esmalte, que é uma estrutura de natureza não remodeladora ${ }^{12}$.

A função ameloblástica, durante essa etapa, é alterada ${ }^{7}$ e, em decorrência da incapacidade de remodelação do esmalte, as alterações durante a formação são permanentemente registradas na superfície dentária ${ }^{22}$. Mediante a determinação da localização do defeito no esmalte, é possível identificar o possível período da vida no qual o distúrbio aconteceu. Existe um período característico de formação de cada grupo dentário. Os dentes decíduos iniciam sua formação durante o período intrauterino e completam seu desenvolvimento vários meses após o nascimento da criança, aproximadamente, no primeiro ano ${ }^{7}$. Nos dentes permanentes, a formação das coroas ocorre após o nascimento e continua até os 6 anos de idade. Durante esses períodos, os dentes são suscetíveis a estímulos que poderão interferir na integridade dentária ${ }^{1}$.

\section{Prevalência}

As prevalências de DDE em crianças com dentição decídua estiveram compreendidas entre $23,9 \%$ e $90,4 \%{ }^{2,5,8,23}$. Em crianças com dentição permanente, a frequência da presença de defeitos de esmalte foi observada em estudos que variaram de $52 \%{ }^{3}$ a $92,1 \%$, $9-11$.

\section{Tipos de defeitos de esmalte}

Clinicamente, são manifestados três tipos de defeitos de esmalte não fluoróticos: as hipoplasias com perda de estrutura dental, as hipoplasias sem perda de estrutura dental e as opacidades (Figura 1).

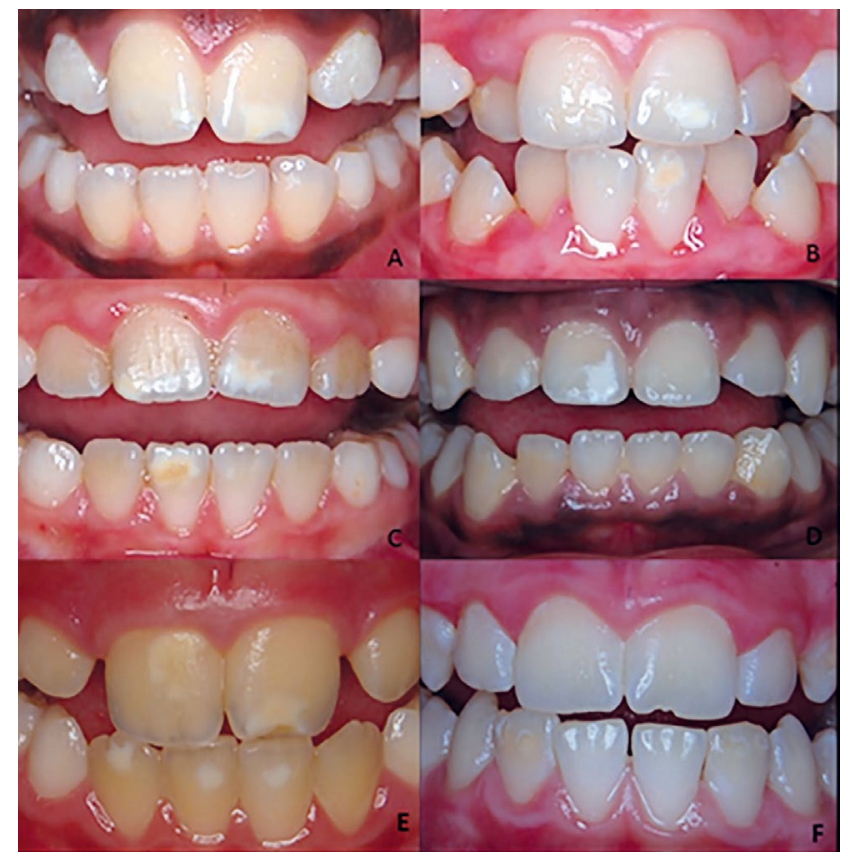

Figura 1 - DDEs observados em escolares: A) opacidades difusas em 12 e 22, opacidade demarcada e opacidade difusa em 21 e 11, B) hipoplasia em 31; opacidade demarcada e difusa em 21 e opacidade difusa em 11 e 41, C) hipoplasia e opacidades difusas em 21 e hipoplasia em 41, D) opacidade demarcada em 11 e opacidades difusas em 21 e 12, E) e F) hipoplasias com perdas de esmalte em espessura

Fonte: arquivo pessoal dos autores.

\section{Hipoplasia}

As alterações que acometem o esmalte durante o estágio de formação da matriz podem resultar na redução da espessura ou da quantidade de esmalte, promovendo a presença de hipoplasias. Os dentes com hipoplasias de esmalte podem apresentar mudança na cor do esmalte para bege, marrom ou amarelo-escuro, sendo que fóssulas, fissuras ou perda maior de algumas áreas de esmalte podem ser observadas $^{22}$.

\section{Opacidades}

Distúrbios ocorridos na fase de maturação ou mineralização da matriz podem levar a deficiências tipo hipocalcificação e, geralmente, manifestam-se como mudanças na translucidez ou opacidades do esmalte. As opacidades são defeitos que apresentam mudanças na coloração, mas sem perda de esmalte $^{13}$.

As opacidades podem ser demarcadas ou difusas. Quando é observada translucidez de grau variável no esmalte, com limites nítidos e coloração esbranquiçada, trata-se de uma opacidade demarcada. A opacidade difusa apresenta-se como uma mancha de coloração esbranquiçada que não apresenta limites nítidos ${ }^{24}$. 


\section{Distribuição}

Os defeitos de desenvolvimento de esmalte podem estar presentes de forma isolada ou combina$\mathrm{da}$, possibilitando visualizar opacidades difusas e delimitadas, hipoplasias e opacidades ou inclusive todos os defeitos em um dente ou em várias áreas da superfície dentária ${ }^{1}$.

As opacidades são os defeitos frequentemente observados na maioria dos estudos, acometendo, principalmente, incisivos e molare ${ }^{16,17,19,25} . \mathrm{Na}$ dentição decídua e permanente, as opacidades de tipo difuso são as mais encontradas ${ }^{4}$. A hipoplasia é o defeito menos comum em ambas as dentições, apresentando prevalências que variam de $0,6 \%^{3}$ até $59,6 \%^{2}$ na dentição decídua e de $0,5 \%^{4}$ até $39 \%^{26}$ na dentição permanente.

\section{Diagnóstico diferencial}

Algumas manchas de diferentes etiologias podem ser confundidas com manchas brancas e defeitos de esmalte hereditários. O diagnóstico diferencial permite identificar a condição ou a doença mediante um processo de eliminação. É necessária a realização de exame clínico e de anamnese para a determinação do diagnóstico final, a fim de selecionar tratamentos adequados mais específicos (Tabela 1 ).

Tabela 1 -Características clínicas para a determinação do diagnóstico diferencial de DDE

\begin{tabular}{|c|c|c|c|c|c|c|c|c|}
\hline Tipo & Cor & Brilho & Forma & Limites & $\begin{array}{l}\text { Integridade } \\
\text { estrutural }\end{array}$ & Localização & Distribuição & $\begin{array}{l}\text { Histórico } \\
\text { familiar }\end{array}$ \\
\hline Hipoplasia & $\begin{array}{l}\text { Branco, marrom, } \\
\text { amarelo }\end{array}$ & Opaca & Assimétrica & Delimitado & Perda parcial & $\begin{array}{l}\text { Vestibular dos } \\
\text { incisivos superiores }\end{array}$ & Bilaterais & Não \\
\hline $\begin{array}{l}\text { Opacidades } \\
\text { difusas }\end{array}$ & Branco & Opaca & Assimétrica & $\begin{array}{l}\text { Não } \\
\text { delimitado }\end{array}$ & Preservada & Vestibular & Bilaterais & Não \\
\hline $\begin{array}{l}\text { Opacidades } \\
\text { demarcadas }\end{array}$ & $\begin{array}{l}\text { Branco, amarelo, } \\
\text { castanho }\end{array}$ & Opaca & Simétrica & Delimitado & Preservada & Vestibular & Bilaterais & Não \\
\hline $\begin{array}{l}\text { Amelogênese } \\
\text { imperfeita }\end{array}$ & $\begin{array}{l}\text { Marrom, amarelo, } \\
\text { castanho-escuro }\end{array}$ & Opaca & $\begin{array}{l}\text { Crateriforme, } \\
\text { áspera, lisa }\end{array}$ & - & $\begin{array}{l}\text { Perda parcial } \\
\text { ou total }\end{array}$ & $\begin{array}{l}\text { Vestibular proximal } \\
\text { palatino }\end{array}$ & Todos & Sim \\
\hline $\begin{array}{l}\text { Mancha } \\
\text { branca }\end{array}$ & $\begin{array}{l}\text { Branco, } \\
\text { amarelo-escuro }\end{array}$ & $\begin{array}{l}\text { Opaca, } \\
\text { brilhante }\end{array}$ & Assimétrica & Delimitado & Preservada & $\begin{array}{l}\text { Cervical, proximal } \\
\text { ou oclusal }\end{array}$ & $\begin{array}{l}\text { Alguns dentes } \\
\text { em erupção }\end{array}$ & Não \\
\hline Fluorose & Branco & Opaca & $\begin{array}{l}\text { Simétrica } \\
\text { linear }\end{array}$ & $\begin{array}{l}\text { Não } \\
\text { delimitado }\end{array}$ & $\begin{array}{l}\text { Preservada, } \\
\text { perda parcial }\end{array}$ & Vestibular & Todos & Não \\
\hline $\begin{array}{l}\text { Mancha por } \\
\text { tetraciclina }\end{array}$ & $\begin{array}{l}\text { Marrom, amarelo, } \\
\text { cinza }\end{array}$ & Brilhante & Linear & $\begin{array}{l}\text { Não } \\
\text { delimitado }\end{array}$ & Preservada & $\begin{array}{l}\text { Dentes anteriores, } \\
\text { cervical }\end{array}$ & Todos & Não \\
\hline
\end{tabular}

Fonte: dados da pesquisa.

\section{Fluorose}

A fluorose é um tipo de hipomineralização do esmalte dentário, relacionada com o consumo anormal de flúor. Clinicamente, na forma menos severa, linhas brancas finas aparecem na superfície dentária de todos os dentes. Em conjunto, formam uma mancha que apresenta um aspecto opaco e calcário ${ }^{14}$. Nas formas mais severas, pode ocorrer a perda do esmalte, levando ao aparecimento de depressões no dente, apresentando pigmentação de amarela a castanho-escura ${ }^{14}$. O período crítico de suscetibilidade à fluorose dentária é durante o segundo e o terceiro anos de vida, quando os dentes estão se formando. O grau de severidade da fluorose dentária depende da dose de flúor ingerida, do tempo de exposição e em qual fase de amelogênese o dente está27.

\section{Mancha branca de cárie}

A mancha branca é a primeira evidência visível de atividade cariogênica na superfície do tecido dentário, causada pela desmineralização por ação dos produtos bacterianos provenientes do biofilme dentário ${ }^{16}$. A lesão no esmalte aparece com um aspecto branco e opaco evidente ou visível após a secagem. Dependendo da atividade da lesão, a superfície pode ser rugosa ou lisa, brilhante ou opaca. Usualmente, está associada à presença de placa e com histórico prévio de cárie ${ }^{15}$. São mais prevalentes na margem gengival vestibular, especialmente em molares, em dentes em erupção ou com maloclusão e superfícies retentivas. As manchas inativas podem migrar até o terço médio da superfície vestibular ${ }^{12,16}$.

\section{Amelogênese}

Amelogênese imperfeita é uma doença hereditária que causa defeitos na formação do esmalte dental de tipo quantitativo, mostrando heterogeneidade clínica e genética ${ }^{28}$. Clinicamente, existem três formas: hipoplásica, hipocalcificada e hipomaturada. Na hipoplásica, não existe a matriz de esmalte adequadamente formada, o esmalte pode ter pouca espessura e/ou fossas e canaletas. Na amelogênese hipocalcificada, a matriz tem espessura normal, 
mas a calcificação é deficiente. Clinicamente, o esmalte não resistente é opaco e branco-amarelado. $\mathrm{Na}$ forma hipomaturada, há um defeito na maturação da estrutura cristal do esmalte, deixando o esmalte com textura amolecida ${ }^{27}$. Os dentes afetados podem apresentar alterações na forma assim como mudanças de cor evidentes, de branco opaco a marrom e/ou amarelado ${ }^{27}$. Usualmente, há perda parcial do esmalte em vários ou na maioria dos dentes. Os dentes afetados podem ficar sensíveis ou propensos à desintegração. É característico o relato de antecedente familiar de situações similares ${ }^{28}$.

\section{Manchas por tetraciclina}

As moléculas da tetraciclina, quando ingeridas na fase de formação dentária, podem ser incorporadas à dentina, gerando manchamento dentário. Clinicamente, observam-se manchas que variam de amarelo ou cinza-claro até marrom-escuro, comumente, na maioria dos dentes. O diagnóstico dessa alteração de esmalte apresenta como parte da anamnese a história de consumo do medicamento ${ }^{27}$.

\section{Fatores associados}

Os defeitos do esmalte dentário têm sido associados a fatores sistêmicos, genéticos, locais e ambientais ${ }^{18}$.

\section{Fatores sociodemográficos}

\section{Renda}

Alguns fatores sociais podem aumentar a predisposição da presença de DDE em crianças ${ }^{6}$. A baixa renda pode afetar o desenvolvimento de estruturas do organismo, como os dentes, podendo estar relacionada com a maior prevalência de DDE em crianças $^{6,29}$. Robles et al. ${ }^{3}(2013)$ observaram a associação de maior prevalência de DDE em indivíduos com nível socioeconômico médio-baixo e baixo e que frequentam escola pública.

O estudo de Ford et al. ${ }^{30}$ (2009) observou que o baixo nível socioeconômico aumentou a predisposição da presença de hipoplasia do esmalte. Porém, Li et al. ${ }^{8}(1995)$ e Slayton et al. ${ }^{31}(2000)$ não encontraram relação entre o perfil socioeconômico da criança e a presença de DDE.

As crianças de famílias de baixa renda, geralmente, estão sujeitas a problemas nutricionais e à maior incidência de infecções, fatores relacionados aos defeitos de esmalte ${ }^{7}$. Essa associação é explicada, também, devido à falta de acompanhamento pré-natal, observada em gestantes de baixa renda, o que pode estar relacionado com a prematuridade do parto ${ }^{6}$.

\section{Idade materna}

A presença de DDE tem sido associada com a idade materna no momento do parto. $\mathrm{Na}$ dentição decídua, a prevalência de DDE foi maior em crianças com mães de idade inferior a 24 anos ao nascimento da criança. A gravidez na adolescência está associada a um maior risco de resultados adversos, incluindo parto prematuro e baixo peso ao nascer?

\section{Fatores relacionados à gestação}

\section{Tabagismo na gestação}

Foi detectada uma relação entre o uso de tabaco durante a gravidez e o aumento da prevalência de hipoplasia na criança. Essa associação pode ser explicada pelo fato de que o uso do tabaco durante a gravidez está intrinsecamente relacionado com o baixo peso ao nascer. Assim, as três variáveis (tabagismo, baixo peso ao nascer e DDE) estão implicadas e inter-relacionadas ${ }^{2}$.

\section{Uso de medicamentos}

Outro fator a ser considerado nos estudos de DDE é o uso de medicamentos pela mãe durante a gestação. Segundo Jacobsen et al. ${ }^{32}$ (2013), crianças cujas mães usaram drogas antiepilépticas durante a gestação tiveram aumento da prevalência de DDE. Drogas antiepilépticas, como o ácido valproico e a oxcarbazepina, induzem à diminuição da densidade óssea, ao risco aumentado de fraturas e a baixos níveis de cálcio no sangue, que, juntamente com outros minerais, é fundamental para o desenvolvimento do esmalte mineralizado ${ }^{32}$.

\section{Doenças na gestação}

Caixeta e Corrêa ${ }^{33}$ (2005) observaram a relação entre a saúde das mães durante a gestação e DDE da criança, associando a hipertensão materna com a presença de DDE. Outras doenças maternas, porém, com menor frequência, foram associadas, tais como diabetes $(5,71 \%)$, eclampsia $(8,57 \%)$, esclerodermia $(2,86 \%)$, deslocamento prematuro da placenta $(2,86 \%)$, anemia $(2,86 \%)$ e cardiopatias $(2,86 \%)^{33}$.

Essas relações podem ser explicadas porque doenças maternas durante a gestação podem levar à prematuridade no nascimento, fator que leva ao baixo peso ao nascer, que é um grande determinante da presença de $\mathrm{DDE}^{33}$. De Oliveira Melo et al. ${ }^{34}$ (2012) encontraram que aspectos relacionados à saúde materna durante a etapa gestacional não estiveram associados à presença de DDE em crianças, embora tenham encontrado associação entre DDE e prematuridade. As doenças analisadas no estudo que não foram associadas ao DDE foram icterícia, apneia, RDS, sepse, PCA, HPIV, enterocolitenecrosante, osteopenia da prematuridade, doença bronco-pulmonar leve/grave, hipoglicemia e gastrosquise ${ }^{34}$. 


\section{Partos múltiplos}

Partos múltiplos foram associados à maior prevalência de defeitos de esmalte nas crianças ${ }^{2}$. Velló et al. $^{2}$ (2010) encontraram essa relação no fato de que crianças com baixo peso ao nascer têm grande prevalência de DDE e, normalmente, as crianças nascem com baixo peso em partos múltiplos.

\section{Fatores relacionados com a criança}

\section{Sexo da criança}

Quanto ao sexo da criança, não há consenso na existência de relação entre a variável sexo da criança e a prevalência de DDE. Algumas pesquisas apontaram maior prevalência de DDE em meninos ${ }^{3,6,8}$, outras não evidenciaram essa associação ${ }^{2,26}$. A maior prevalência de defeitos de esmalte em crianças do sexo masculino foi sugerida devido a um aumento nutricional, pelo crescimento mais rápido nos meninos, tornando-os mais suscetíveis à formação de defeitos de esmalte quando comparados às meninas ${ }^{35}$. De outro modo, o maior peso ao nascer, apresentado pelos meninos (média de 3.318,6 kg) quando comparados às meninas (média de $3.182,0 \mathrm{~kg}$ ), aumenta a predisposição deles para a presença de $\mathrm{DDE}^{8}$.

\section{Nascimento a pré-termo/prematuridade}

Estudos realizados em diferentes países revelam que o desenvolvimento de defeitos de esmalte em dentes decíduos está associado a diversos fatores, como a prematuridade e o baixo peso no nascimento ${ }^{5,16}$

A prematuridade da criança foi relacionada à presença de defeitos de esmalte nos dentes decíduos $^{8}$. Crianças nascidas antes de 37 semanas de gestação foram 2,6 vezes mais suscetíveis a serem afetadas por algum tipo de DDE quando comparadas a crianças maturas 5 .

Crianças nascidas a pré-termo, que precisaram de intubação orotraqueal e de ventilação mecânica após o parto, apresentaram maior prevalência de $\mathrm{DDE}^{16,25,34}$. A intubação pode promover algum efeito traumático sobre o esmalte do dente em formação ${ }^{16,25,34}$. Nas crianças com histórico de intubação, 0 defeito mais encontrado foi a hipoplasia, no incisivo central superior direito e incisivo lateral superior esquerdo, fato que pode ocorrer devido ao atrito do laringoscópio com a gengiva ${ }^{34}$.

\section{Baixo peso ao nascer}

O baixo peso ao nascer das crianças foi associado com a maior prevalência de DDE em relação àquelas que apresentaram peso normal ao nascimento ${ }^{2,6,8,36}$. Em relação ao baixo peso do recém-nascido, alguns estudos observaram que os defeitos de esmalte mais encontrados foram as opacidades demarcadas nos incisivos e molares ${ }^{16,36,37}$.
Entretanto, no estudo de Masumo et al. ${ }^{35}$ (2013), baixo peso ao nascer foi associado apenas à maior prevalência de hipoplasia.

\section{Aleitamento materno}

A ausência de amamentação foi relacionada a defeitos no esmalte ${ }^{8}$. Crianças não amamentadas foram 3,2 vezes mais suscetíveis a serem afetadas por DDE quando comparadas com aquelas que foram amamentadas ${ }^{5,37,38}$. A ausência de amamentação é um importante fator predisponente ao aparecimento de DDE, porque faz com que a criança seja privada de substâncias nutricionais, como a vitamina $\mathrm{D}$, necessárias para o seu desenvolvimento e, consequentemente, para a formação do esmalte ${ }^{8}$.

O leite materno é o primeiro veículo de oferta de nutrientes para o indivíduo, entretanto, nem sempre garante uma nutrição completa do neonato. A deficiência de cálcio está relacionada à hipoplasia do esmalte em crianças nascidas prematuramente abaixo do peso normal (menos de 2.500 gramas) ${ }^{37,38}$.

\section{Má nutrição}

Deficiências nutricionais nos primeiros períodos de desenvolvimento da maturação dentária podem levar a alterações no esmalte e também na sequência eruptiva. A deficiência de cálcio e vitaminas pode resultar em hipoplasia do esmalte por distúrbios na função secretora dos ameloblastos ${ }^{29}$.

\section{Sofrimento fetal}

Crianças que apresentaram baixos valores de Apgar assim como problemas de nutrição parietal também apresentaram maior prevalência de defeitos de esmalte ${ }^{2}$. O índice de Apgar avalia as condições vitais dos recém-nascidos. A relação entre baixo valor de Apgar e maior prevalência de DDE pode estar relacionada com a alteração da função das células ameloblásticas devido à privação de oxigênio, comprometendo o correto desenvolvimento do esmalte dentário ${ }^{2}$.

\section{História de doenças na infância}

Doenças sistêmicas em crianças podem aumentar a chance de presença de defeitos no esmalte do dente permanente. Crianças com dentição permanente, alta incidência de infecções na infância e com problemas nutricionais, relação ao desenvolvimento dos dentes, podem apresentar defeitos de esmalte ${ }^{6,29}$. Pacientes pediátricos com asma grave, de início precoce, têm um risco aumentado de defeitos do esmalte dentário ${ }^{18}$. Quando esse ponto é levado em consideração, os defeitos mais encontrados são as opacidades difusas e demarcadas ${ }^{19}$. Crianças com histórias de infecções respiratórias ou catapora, expostas à fumaça de cigarro, com infecções do trato urinário e otite estão predispostas a hipoplasia do esmalte ${ }^{30}$. 
No estudo de Guergolette et al. ${ }^{18}$ (2009), dos pacientes do grupo asma, $61(89,7 \%)$ apresentaram defeitos do esmalte dentário, em comparação com apenas $26(38,2 \%)$ daqueles do grupo controle. Conforme Wogelius et al. ${ }^{19}$ (2010), existe uma tendência à associação entre medicamentos inalados para asma e severas opacidades demarcadas, que resultam em perda macroscópica de substância dentária e, possivelmente, em necessidade de um cuidado restaurador. Isso poderia estar associado com a asma propriamente dita, com a gravidade da asma, ou pode ser um efeito de corticosteroides ou da combinação de b2-agonistas (remédios inalados para controle da asma) e de corticosteroides ${ }^{19}$.

Crianças com doença celíaca apresentaram $42,2 \%$ de DDE, enquanto crianças sem a doença apresentaram $9,4 \%$. Isso ocorre porque o dano intestinal leva à má absorção de vitamina $\mathrm{D}$ em pacientes com doença celíaca, e uma possível explicação para a presença de defeitos de esmalte nessas crianças é que a hipoplasia do esmalte é causada pela hipocalcemia ${ }^{17}$.

Outras causas sistêmicas frequentemente relacionadas são: infecções do trato respiratório, otite média, infecções do trato urinário, complicações osmóticas, deficiências nutricionais (vitaminas $\mathrm{A}, \mathrm{C} \mathrm{e}$ $\mathrm{D}$, cálcio e fósforo), doenças exantemáticas (sarampo, varicela, escarlatina, rubéola), sífilis congênita, doença celíaca, prematuridade do nascimento, causas idiopáticas, entre outros motivos ${ }^{39}$. Nesses casos, a maior presença de hipoplasias explica-se pelo fato de os ameloblastos constituírem um dos grupos de células mais sensíveis do corpo, no que diz respeito à função metabólica. Entretanto, a hipoplasia de esmalte só aparece se a injúria ocorrer na época em que os dentes estiverem em desenvolvimento ou, mais especificamente, durante a fase da formação do esmalte. Depois de estar mineralizado, não existe mais risco de ocorrer esses defeitos.

\section{Cárie na dentição primária}

Há controvérsias em relação à associação entre cárie e DDE. Na dentição decídua, estudos observaram a associação entre a presença de cárie e defeitos de esmalte, em que a progressão das lesões de cárie ocorreria mais rapidamente em dentes com $\mathrm{DDE}^{8,15,16}$. Situação similar é observada na dentição permanente. Estudos mostraram que crianças com DDE tiveram maior prevalência de cárie ${ }^{12,14}$. Porém, outros estudos não registraram tal associação, tanto na dentição decídua ${ }^{25}$ quanto na permanente ${ }^{2}$.

A hipótese de $\mathrm{Li}$ et al. ${ }^{8}$ (1995) é de que especialmente no caso das hipoplasias permitir-se-ia a aderência das bactérias cariogênicas, facilitando o surgimento de cárie. Seow ${ }^{23}$ (1991) sugeriu que os defeitos de esmalte fazem com que o dente torne-se menos resistente à cárie, devido à presença de imperfeições no esmalte.

\section{Trauma dentário na dentição primária}

Crianças que sofreram traumas dentários nos decíduos apresentaram defeitos de esmalte nos dentes permanentes ${ }^{10,40}$. Isso pode ser explicado pelo fato de que o deslocamento traumático de raízes decíduas afeta o desenvolvimento de germes permanentes, alterando os ameloblastos da fase secretora $^{40}$.

Alguns estudos observaram a relação entre a idade da criança e a possibilidade de trauma, indicando que crianças mais jovens apresentam maior risco de trauma e, assim, maior risco de surgimento de DDE ${ }^{10}$. Porém, segundo Do Espirito Santo et al. ${ }^{11}$ (2009), não houve relação entre a idade da criança no momento do trauma e o surgimento de DDE. No entanto, houve relação significativa entre traumas da dentição decídua e DDE na dentição permanente. Dentre os DDEs, o que mais esteve relacionado nessa situação foi a hipoplasia. Lesões traumáticas no dente decíduo combinadas à idade da criança no momento do acidente podem indicar danos na dentição secundária, o que pode ocorrer em $50 \%$ dos incidentes $^{41}$.

\section{Presença de defeitos de esmalte na dentição decídua}

A presença de defeitos de esmalte na dentição decídua é associada à ocorrência de defeitos na dentição permanente, com chance de ocorrer duas vezes maior $^{26}$.

\section{Discussão}

O DDE é um distúrbio no desenvolvimento dental, em que a função ameloblástica é prejudicada, acarretando alterações na estrutura do esmalte dental ${ }^{7}$. Estudos relataram prevalências que podem variar de $23,9 \%$ a $90,4 \%$ na dentição decídua ${ }^{2,5,8,23}$ e de $52 \%$ a $92,1 \%$ na permanente $e^{4,9-11}$. Os fatores que a desencadeiam estão relacionados principalmente a injúrias durante o desenvolvimento da estrutura dental ${ }^{1}$. Na dentição decídua, traumas durante a gestação e o consumo de medicamentos podem desencadear tal distúrbio, enquanto que na dentição permanente eventos traumáticos ou lesões de cárie nos correspondentes decíduos estão fortemente associados com DDE $\mathrm{DD}^{2,5,6}$.

Quando esses distúrbios ocorrem durante a fase de formação da matriz do esmalte, menor espessura ou quantidade de esmalte é depositada, e isso resulta em manchas clinicamente observadas com colorações que variam do branco-bege ao amarelo-escu$\mathrm{ro}^{13}$. No entanto, quando esses distúrbios ocorrem durante a fase de maturação ou mineralização, observamos uma hipocalcificação dos tecidos já depositados, que se caracterizam clinicamente com manchamentos opacos que interferem na translucidez do esmalte, podendo ser difusos ou demarcados ${ }^{24}$. 
A determinação da etiologia das manchas torna-se uma fase clínica importante, pois as manchas provenientes de defeitos de desenvolvimento de esmalte não fluoróticos podem ser confundidas com outros manchamentos presentes no elemento dental. A presença de manchas de diversas etiologias pode ser encontrada ao mesmo tempo, dificultando ainda mais o diagnóstico clínico. As manchas de cárie em estágio inicial ativas ou inativas podem ser confundidas com os DDEs. Manchas de cárie são esbranquiçadas, localizadas geralmente em cervical e associadas à placa. Torna-se importante frisar que estudos encontram frequentemente a associação entre a presença de defeitos de esmalte e a presença de cárie dentária ${ }^{42}$.

Em situações mais severas, pode existir sobreposição, prevalecendo o efeito destrutivo da cárie e influenciando a qualidade de vida das crianças ${ }^{43}$. Estudos demonstram que as hipoplasias favorecem a adesão de bactérias e o surgimento da cárie dental, situação que poderá ser agravada devido ao esmalte com defeito ser menos resistente à ação dos ácidos provenientes das bactérias ${ }^{23}$.

Já a fluorose dentária, caracterizada por finas linhas esbranquiçadas, opacas, quando branda, ou acastanhadas, quando severa ${ }^{14}$, facilmente promove confusão diagnóstica. Por estar relacionada ao consumo excessivo de flúor durante os estágios de formação, ela aparece em todos os elementos e superfícies dentais formadas no mesmo período, por serem afetados da mesma forma durante o seu desenvolvimento ${ }^{27}$.

$\mathrm{O}$ manchamento permanentemente produzido pela tetraciclina pode ser incorporado à dentina quando ela está em formação, ocasionando manchas amarelas ou cinza-claras, até colorações mais escuras, como marrom ${ }^{27}$. O relato da ingestão de tal medicamento na infância, durante a anamnese realizada pelos profissionais, é complemento fundamental para determinar a etiologia da mancha.

A amelogênese imperfeita é outra alteração na formação do esmalte dental que apresenta um componente genético comprovado ${ }^{28}$. Por ser uma anomalia que pode ocorrer tanto nos processos de deposição, maturação ou calcificação, pode ser expressa clinicamente como manchas branco-amareladas, opacas, ou amarelas e marrons ${ }^{27}$, frequentemente associadas à perda parcial do esmalte dental com textura amolecida, em diversos formatos irregulares desorganizados ou sequências em forma de fossas ou canaletas. Tais alterações podem também facilitar a progressão de lesões de cárie ${ }^{12}$. Dessa forma, a anamnese permitirá complementar o diagnóstico clínico, assim como a localização de populações em risco para poder implementar medidas de prevenção da ocorrência de maior perda estrutural dentária.

Além disso, diversos fatores (sistêmicos e ambientais) foram associados com o DDE. Aspectos sociais, como a renda, parecem aumentar a predis- posição dos indivíduos a apresentar DDE ${ }^{29}$. Estudos que avaliaram crianças em condições socioeconômicas desfavoráveis mostraram que elas apresentam maior prevalência de $\mathrm{DDE}^{3}$, isso pode ser explicado por essas pessoas estarem expostas a problemas nutricionais durante a infância. Carências nutricionais durante os períodos de desenvolvimento podem levar a distúrbios na função dos ameloblastos, acarretando hipoplasias ${ }^{29}$. Ainda, tais crianças estão sujeitas a maiores índices de infecções, que também são fatores predisponentes para $\mathrm{DDE}^{7}$.

Filhos de gestantes que têm o hábito de consumir tabaco durante a gravidez também podem apresentar maior incidência de $\mathrm{DDE}^{2}$, e isso está relacionado com baixo peso ao nascimento ${ }^{6}$. Essas crianças, em geral, acabam apresentando deficiências nutricionais e, consequentemente, DDE. Doenças durante a gestação, como hipertensão materna, diabetes e pré-eclampsia, são relacionadas com a presença de DDE por poderem levar a partos prematuros e, por conseguinte, ao baixo peso ao nascer ${ }^{33}$.

Tais acontecimentos estão intrinsecamente inter-relacionados e podem acabar desencadeando alterações no esmalte dental. Crianças que apresentaram doenças durante a infância, como infecções respiratórias e no trato urinário, catapora, ou foram expostas à fumaça do cigarro tendem a apresentar prevalência maior de $\mathrm{DDE}^{30}$. Esses acontecimentos, que podem ocorrer tanto na vida intrauterina quanto na infância, são relatados como fatores de risco que podem desencadear alterações no desenvolvimento do esmalte dental.

Dessa forma, a presença de defeitos de esmalte pode promover o aparecimento e a progressão de cárie, o que pode ocasionar perda precoce de estrutura dentária e, como consequência, sensibilidade dentária, maloclusão e problemas estéticos, que influenciam na qualidade de vida do sujeito. Além disso, essas consequências marcam etapas de distúrbios durante o desenvolvimento dos indivíduos, que podem ser determinantes na futura saúde bucal e geral.

\section{Considerações finais}

A presente revisão mostrou que, nas dentições decídua e permanente, a presença de DDE em crianças é comum e está associada a diversos fatores. O DDE e a cárie dentária apresentam-se associados. Dessa forma, o diagnóstico adequado é necessário para a determinação de tratamento e conduta adequados. 


\section{Abstract}

Objective: The present review analyzed the clinical and epidemiological characteristics of enamel development in children with permanent and primary dentition. Literature review: Non-fluorotic developmental enamel defects are flaws produced in the internal structure of enamel during its development. Developmental enamel defects may be found in both dentitions with prevalence ranging from $23.9 \%$ to $90.4 \%$ in primary dentition and $52 \%$ to $92.1 \%$ in permanent dentition. These enamel disorders may be associated with several factors related to children and maternal characteristics. The presence of developmental enamel defects may be an indication of previous pathological events. Depending on the severity, these defects may predispose teeth to other dental problems such as caries or tooth wear. If the defect is severe, esthetic dental problems, and consequently, psychological problems may interfere with the quality of life of children. Severe defects may lead to the search for more invasive esthetic treatments, promoting early dental structure loss. Final considerations: The knowledge of the etiology and clinical characteristics of developmental enamel defects is indispensable for the formulation of an adequate treatment plan.

Keywords: Developmental enamel defects. Primary dentition. Permanent dentition. Associated factors.

\section{Referências}

1. Correa-Faria P, Martins-Junior PA, Vieira-Andrade RG, Oliveira-Ferreira F, Marques LS, Ramos-Jorge ML. Developmental defects of enamel in primary teeth: prevalence and associated factors. Int J Paediatr Dent 2013; 23(3):173-9.

2. Vello MA, Martinez-Costa C, Catala M, Fons J, Brines J, Guijarro-Martinez R. Prenatal and neonatal risk factors for the development of enamel defects in low birth weight children. Oral Dis 2010; 16(3):257-62.

3. Robles MJ, Ruiz M, Bravo-Perez M, Gonzalez E, Penalver MA. Prevalence of enamel defects in primary and permanent teeth in a group of schoolchildren from Granada (Spain). Med Oral Patol Oral Cir Bucal 2013; 18(2):e187-93.

4. Wong HM, McGrath C, King NM. Diffuse opacities in 12-year-old Hong Kong children - four cross-sectional surveys. Community Dent Oral Epidemiol 2014; 42(1):61-9.

5. Lunardelli SE, Peres MA. Prevalence and distribution of developmental enamel defects in the primary dentition of preschool children. Braz Oral Res 2005; 19(2):144-9.

6. Massoni AC, Chaves AM, Rosenblatt A, Sampaio FC, Oliveira AF. Prevalence of enamel defects related to pre-, peri- and postnatal factors in a Brazilian population. Community Dent Health 2009; 26(3):143-9.

7. Correa-Faria P, Martins-Junior PA, Vieira-Andrade RG, Oliveira-Ferreira F, Marques LS, Ramos-Jorge ML. Developmental defects of enamel in primary teeth: prevalence and associated factors. Int J Paediatr Dent 2013; 23(3):173-9.

8. Li Y, Navia JM, Bian JY. Prevalence and distribution of developmental enamel defects in primary dentition of Chinese children 3-5 years old. Community Dent Oral Epidemiol $1995 ; 23(2): 72-9$
9. Carvalho JC, Silva EF, Gomes RR, Fonseca JA, Mestrinho HD. Impact of enamel defects on early caries development in preschool children. Caries Res 2011; 45(4):353-60.

10. Christophersen P, Freund M, Harild L. Avulsion of primary teeth and sequelae on the permanent successors. Dent Traumatol 2005; 21(6):320-3.

11. do Espirito Santo Jacomo DR, Campos V. Prevalence of sequelae in the permanent anterior teeth after trauma in their predecessors: a longitudinal study of 8 years. Dent Traumatol 2009; 25(3):300-4.

12. Farsi N. Developmental enamel defects and their association with dental caries in preschoolers in Jeddah, Saudi Arabia. Oral Health Prev Dent 2010; 8(1):85-92.

13. Hoffmann RH, de Sousa Mda L, Cypriano S. Prevalence of enamel defects and the relationship to dental caries in deciduous and permanent dentition in Indaiatuba, Sao Paulo, Brazil. Cad Saude Publica 2007; 23(2):435-44.

14. Oliveira AF, Chaves AM, Rosenblatt A. The influence of enamel defects on the development of early childhood caries in a population with low socioeconomic status: a longitudinal study. Caries Res 2006; 40(4):296-302.

15. Targino AG, Rosenblatt A, Oliveira AF, Chaves AM, Santos VE. The relationship of enamel defects and caries: a cohort study. Oral Dis 2011; 17(4):420-6.

16. Nelson S, Albert JM, Lombardi G, Wishnek S, Asaad G, Kirchner HL, et al. Dental caries and enamel defects in very low birth weight adolescents. Caries Res 2010; 44(6):509-18.

17. Avsar A, Kalayci AG. The presence and distribution of dental enamel defects and caries in children with celiac disease. Turk J Pediatr 2008; 50(1):45-50.

18. Guergolette RP, Dezan CC, Frossard WT, Ferreira FB, Cerci Neto A, Fernandes KB. Prevalence of developmental defects of enamel in children and adolescents with asthma. J Bras Pneumol 2009; 35(4):295-300.

19. Wogelius P, Haubek D, Nechifor A, Norgaard M, Tvedebrink T, Poulsen S. Association between use of asthma drugs and prevalence of demarcated opacities in permanent first molars in 6-to-8-year-old Danish children. Community Dent Oral Epidemiol 2010; 38(2):145-51.

20. Hong L, Levy SM, Warren JJ, Broffitt B. Association between enamel hypoplasia and dental caries in primary second molars: a cohort study. Caries Res 2009; 43(5):345-53.

21. Vargas-Ferreira F, Ardenghi TM. Developmental enamel defects and their impact on child oral health-related quality of life. Braz Oral Res 2011; 25(6):531-7.

22. Seow WK. A study of the development of the permanent dentition in very low birthweight children. Pediatr Dent 1996; 18(5):379-84

23. Seow WK. Enamel hypoplasia in the primary dentition: a review. ASDC J Dent Child 1991; 58(6):441-52.

24. A review of the developmental defects of enamel index (DDE Index). Commission on Oral Health, Research \& Epidemiology. Report of an FDI Working Group. Int Dent J 1992; 42(6):411-26

25. Cruvinel VR, Gravina DB, Azevedo TD, Rezende CS, Bezerra AC, Toledo OA. Prevalence of enamel defects and associated risk factors in both dentitions in preterm and full term born children. J Appl Oral Sci 2012; 20(3):310-7.

26. Seow WK, Ford D, Kazoullis S, Newman B, Holcombe T. Comparison of enamel defects in the primary and permanent dentitions of children from a low-fluoride District in Australia. Pediatr Dent 2011; 33(3):207-12. 
27. Passos IA, Costa JDMC, Melo JM, Forte FDS, Sampaio FC. Defeitos do esmalte: etiologia, características clínicas e diagnóstico diferencial. Rev Inst Ciênc Saúde 2007; 25(2):187-92.

28. Gadhia K, McDonald S, Arkutu N, Malik K. Amelogenesis imperfecta: an introduction. Br Dent J 2012; 212(8):377-9.

29. Rugg-Gunn AJ, Al-Mohammadi SM, Butler TJ. Malnutrition and developmental defects of enamel in 2- to 6-year-old Saudi boys. Caries Res 1998; 32(3):181-92.

30. Ford D, Seow WK, Kazoullis S, Holcombe T, Newman B. A controlled study of risk factors for enamel hypoplasia in the permanent dentition. Pediatr Dent 2009; 31(5):382-8.

31. Slayton RL, Warren JJ, Kanellis MJ, Levy SM, Islam M. Prevalence of enamel hypoplasia and isolated opacities in the primary dentition. Pediatr Dent 2001; 23(1):32-6.

32. Jacobsen PE, Henriksen TB, Haubek D, Ostergaard JR. Developmental enamel defects in children prenatally exposed to anti-epileptic drugs. PLoS One 2013; 8(3):e58213.

33. Caixeta FF, Corrêa MSNP. Os defeitos do esmalte e a erupção dentária em crianças prematuras. Rev Assoc Med Bras 2005; 51(4):195-9.

34. de Oliveira Melo NS, da Silva RP, de Lima AA. The neonatal intubation causes defects in primary teeth of premature infants. Biomed Pap Med Fac Univ Palacky Olomouc Czech Repub 2014; 158(4):605-12.

35. Masumo R, Bardsen A, Astrom AN. Developmental defects of enamel in primary teeth and association with early life course events: a study of 6-36 month old children in Manyara, Tanzania. BMC Oral Health 2013; 13:21.

36. Franco KM, Line SR, de Moura-Ribeiro MV. Prenatal and neonatal variables associated with enamel hypoplasia in deciduous teeth in low birth weight preterm infants. J Appl Oral Sci 2007; 15(6):518-23.

37. Correa-Faria P, Martins-Junior PA, Vieira-Andrade RG, Marques LS, Ramos-Jorge ML. Perinatal factors associated with developmental defects of enamel in primary teeth: a case-control study. Braz Oral Res 2013; 27(4):363-8.

38. Lunardelli SE, Peres MA. Breast-feeding and other motherchild factors associated with developmental enamel defects in the primary teeth of Brazilian children. J Dent Child (Chic) 2006; 73(2):70-8

39. Hall RK. Prevalence of developmental defects of tooth enamel (DDE) in a pediatric hospital department of dentistry population (1). Adv Dent Res 1989; 3(2):114-9.

40. Altun C, Cehreli ZC, Guven G, Acikel C. Traumatic intrusion of primary teeth and its effects on the permanent successors: a clinical follow-up study. Oral Surg Oral Med Oral Pathol Oral Radiol Endod 2009; 107(4):493-8.

41. Carvalho V, Jacomo DR, Campos V. Frequency of intrusive luxation in deciduous teeth and its effects. Dent Traumatol 2010; 26(4):304-7.

42. Vargas-Ferreira F, Salas MM, Nascimento GG, Tarquinio SB, Faggion CM Jr, Peres MA, et al. Association between developmental defects of enamel and dental caries: A systematic review and meta-analysis. J Dent 2015; 43(6):619-28.

43. Vargas-Ferreira F, Zeng J, Thomson WM, Peres MA, Demarco FF. Association between developmental defects of enamel and dental caries in schoolchildren. J Dent 2014; 42(5):540-6.
Endereco para correspondência:

Luiz Alexandre Chisini

Faculdade de Odontologia

Universidade Federal de Pelotas

Rua Gonçalves Chaves, 457, Sala 501

96015-560 Pelotas, RS

Telefone: (53) 98112-1141

E-mail: luizalexandrechisini@hotmail.com

Recebido: 30/09/2015. Aceito: 02/06/2016. 